\title{
Isolation of polyphenols from the extracts of olive leaves (Olea europaea L.) by adsorption on silk fibroin
}

\author{
Evren Altıok, Deniz Bayçın ${ }^{1}$, Oguz Bayraktar*, Semra Ülkü \\ Biochemical Engineering Research Laboratory (BERL), Department of Chemical Engineering, \\ Izmir Institute of Technology, Gülbahçe Köyü, 35430, Urla-Izmir, Turkey \\ Received 17 August 2007; received in revised form 15 January 2008; accepted 17 January 2008
}

\begin{abstract}
General objective of the present work was to assess the isolation of polyphenols from olive leaves. The effects of extraction conditions on the total phenol content and antioxidant activity of olive leaf extract (OLE) were investigated. An extract with good antioxidant activity (7.52 mmol of Trolox equivalent antioxidant capacity (TEAC)/g olive leaf extract), and a high content of oleuropein (13.4\%) and rutin (0.18\%) could be obtained using $70 \%$ ethanol as extraction solvent. There was a good correlation between the antioxidant activity and the total phenol content. Furthermore, silk fibroin was used as a novel adsorbent to recover the polyphenols from the olive leaf extracts. The adsorbed amounts of rutin and oleuropein were $15 \mathrm{mg}$ rutin/g silk fibroin and $96 \mathrm{mg}$ oleuropein/g silk fibroin. Fraction consisting of mainly oleuropein and fraction rich in rutin, luteolin-7-glucoside, verbascoside, apigenin-7-glucoside were obtained by using silk fibroin filled column. Silk fibroin was found to be a promising adsorbent for the purification of oleuropein and rutin from olive leaf extracts.
\end{abstract}

(C) 2008 Elsevier B.V. All rights reserved.

Keywords: Olive leaves; Oleuropein; Rutin; Antioxidant; Silk fibroin

\section{Introduction}

For centuries plants have provided humankind with useful, sometimes lifesaving, drugs. In the last century, the use of natural medicine has expanded and many researches have been performed on active components of herbs. These components are known as plant secondary metabolites [1] and possess higher antioxidative, antimicrobial, antiviral and anti-inflammatory properties [2-4]. Free radical scavenging properties of these metabolites can be explained by their high antioxidant activities. The primary cause of certain human diseases (e.g. heart and cardiovascular diseases, cancers, aging and immune system related diseases) is thought to be the free radicals. Free radicals are metabolized in human body because of the occupational exposure to chemically and structurally diverse environmental pollutants including pesticides, toxic chemical wastes, air pollutants, radiation [5] and physical stress. Phytochemicals in

\footnotetext{
* Corresponding author. Tel.: +90 232750 6657; fax: +90 2327506645 .

E-mail address: oguzbayraktar@iyte.edu.tr (O. Bayraktar).

${ }^{1}$ Present address: Department of Chemical \& Biomolecular Engineering, Johns Hopkins University, 221Maryland Hall, 3400 North Charles Street, Baltimore, MD 21218, USA.
}

fruits and vegetables act synergistically and additively to provide potential health benefits against chronic diseases by inhibiting the harmful effects of free radicals [2,4-7].

Olive has an important place in the Mediterranean diet because of its physiologically active polyphenols [8,9]. Olive leaf extract (OLE) has also been used by native people of these areas in folk medicine. This property can be linked to the fact that the leaves are rich in polyphenols, especially in oleuropein, rutin, verbacoside, apigenin-7-glucoside and luteolin-7-glucoside [10,11]. The concentration of polyphenolic compounds in olive leaf changes depending on the quality, origin and variety [12] of the plant material. Recent experimental and theoretical studies found that olive leaf extract has anti-HIV activity by blocking the HIV virus entry to host cells [13,14]. Oleuropein is the most abundant polyphenol in olive leaves, which has been used in a number of medical treatments[10]. However, olive leaves are considered as one of the by products of olive oil industry in Turkey. This raw material has low cost and high availability and used as a fuel after drying or as an animal feed.

Recently several studies focused on contents of the olive leaves and extraction of these high-added value compounds from olive leaves [15-21]. Solvent extraction is a favorable 
process since heat sensitive materials can be recovered at low temperatures. For this reason it is preferred for the manufacturing of polyphenol-rich products from plants for their further use in pharmacological, food and cosmetic industry. In order to optimize the recovery of bioactive compounds, the parameters affecting the extraction process should be investigated. Up to now, the use of olive leaves is limited to crude extracts. Further purification is required in order to obtain concentrated specific components selectively since many other compounds such as sugars, protein or metals may exist in the plant extracts [22,23]. Since adsorption is a low cost separation technique, it is preferred for the selective recovery of target plant metabolites from the crude extracts [22]. Synthetic adsorbents have been applied for adsorbing valuable polyphenols from plant extracts [23-29]. Many biopolymers, such as collagen and cellulose have also been used as adsorbents for the recovery of polyphenols and the interaction between the biopolymers and antioxidative polyphenols have been widely investigated [1].

Silk fibroin is an edible protein polymer which has functional aminoacids in its structure, and it is preferred in many biotechnological applications such as drug delivery and tissue engineering [30-32]. Its hydrophobic character makes it favorable adsorbent used in adsorption studies [33]. Because of its promising health effects and bounding mechanisms, silk fibroin can be considered as a potent adsorbent for the isolation of antioxidants from the crude olive leaf extracts [34].

This study aimed to investigate the efficiency of extraction of polyphenols from Turkish olive leaves by using different solvents and their aqueous solutions. The highest yield for extraction process was determined by analyzing the total phenol contents and antioxidant capacities of olive leaf extracts. Furthermore, the potential use of silk fibroin as a novel adsorbent to recover polyphenols from crude olive leaf extracts was investigated.

\section{Materials and methods}

\subsection{Plant material, reagents, and standards}

Fresh green olive leaves were collected from the olive tree grown in the campus of Izmir Institute of Technology (Turkey). Field collections were made in December 2004. High performance liquid chromatography (HPLC) grade ethanol used as extraction solvent was obtained from Riedel-de Haën (Germany). HPLC grade acetonitrile used as mobile phase for HPLC was obtained from Sigma-Aldrich (Germany). HPLC grade acetic acid used as mobile phase for HPLC was obtained from Merck (Germany). Oleuropein, verbascoside, tyrosol, hydroxytyrosol, apigenin-7-glucoside, luteolin-7-glucoside, luteolin, and catechin were kindly provided by Natural Products Research Development Unit (NPRDU) (Turkey). Internal standard coumarin was obtained from Extrasynthese (France). Rutin (98.5\%) was obtained from Merck (Germany). Vanillin, vanillic acid, caffeic acid were purchased from Sigma-Aldrich (Germany). ABTS (2,2'-azinobis(3ethylbenzothiazoline-6-sulphonic acid) from Sigma-Aldrich
(Germany), Trolox (6-hydroxy-2,5,7,8,-tetramethylchroman-2carboxylic acid) and potassium persulfate $\left(\mathrm{K}_{2} \mathrm{O}_{8} \mathrm{H}_{8}\right)$ from Fluka (Germany) were used in antioxidant analysis. Sodium carbonate was purchased from Fluka (Germany). Tannic acid used in total phenol content analysis was obtained from Sigma-Aldrich (Germany) and Folin-Ciocalteau's reagent was provided by Merck (Germany). Silk fibroin used as an adsorbent was purchased from Silk Biochemical Co., LTD. (China).

\subsection{Preparation of olive leaf samples}

Olive leaves were washed with deionized water and dried at $37^{\circ} \mathrm{C}$ for 3 days. Then the dried leaves were pulverized in a blender in order to decrease the particle size of the leaves to $90-150 \mu \mathrm{m}$. Finally the olive leaf powder was stored in light protected glass bottles for further use.

\subsection{Extraction of olive leaf polyphenols}

The solvent type is the most important factor affecting the efficiency of liquid solid extraction. For this reason, different solvents; acetone, ethanol and their aqueous forms (10-90\%, $\mathrm{v} / \mathrm{v}$ ) were investigated to determine the effective extraction of polyphenolic compounds from olive leaf. Deionized water was used in all experiments. After $24 \mathrm{~h}$ extraction time, the extracts were filtrated and centrifuged for $5 \mathrm{~min}$ at $5000 \mathrm{rpm}$. The total phenol contents and antioxidant capacities of all extracts were determined.

In order to obtain dried OLE, the extraction solvent was removed by using rotary evaporator at $38^{\circ} \mathrm{C}$ with a 120 -rpm rotation under vacuum. Then, solvent free OLE was dried by using a freeze drier system at $-52{ }^{\circ} \mathrm{C}$ and $0.2 \mathrm{mbar}$. Dried OLE powder was stored in light protected glasses until further use.

The time required for the effective extraction was determined by taking samples at every $2 \mathrm{~h}$ during $24 \mathrm{~h}$ and analysing these samples for their total phenol contents.

\subsection{Total phenol content analysis}

Total phenol content of extracts was determined by the method Folin-Ciocalteu assay. An aliquot $(0.5 \mathrm{ml})$ of each extract was reacted with the freshly prepared $1.25 \mathrm{ml}$ of $20 \%$ sodium carbonate and $0.5 \mathrm{ml}$ of $1 \mathrm{~N}$ Folin reagent in a screw-capped test tube. Required dilutions were prepared with distilled water. Test tubes were vortexed and after $40 \mathrm{~min}$, absorbance readings were recorded at $725 \mathrm{~nm}$. The phenol content was expressed as mg tannic acid per gram of dry olive leaf.

\subsection{Total antioxidant activity analysis}

The Trolox equivalent antioxidant capacity (TEAC) assessment was performed in terms of radical scavenging ability according to the ABTS $/ \mathrm{K}_{2} \mathrm{~S}_{8} \mathrm{O}_{2}$ method. The $\mathrm{ABTS}^{+}$radical was generated by a reaction between $7 \mathrm{mM}$ ABTS and activated with $2.45 \mathrm{mM} \mathrm{K}_{2} \mathrm{~S}_{8} \mathrm{O}_{2}$. The $\mathrm{ABTS}^{+}$solution was diluted with 
ethanol to an absorbance of $0.70( \pm 0.03)$ at $734 \mathrm{~nm}$ and equilibrated at $30^{\circ} \mathrm{C}$. Twenty micoliters of sample was added to $\mathrm{ABTS}^{+}$solution and absorbance decrease was recorded during $6 \mathrm{~min}$. Series of dilutions were prepared such that they produced between $20 \%$ and $80 \%$ inhibition of the blank absorbance. TEAC calculation was performed by the slope of the plot of the percentage inhibition of absorbance versus concentration plot for the antioxidant in analysis is divided by the slope of the plot for standard; Trolox. The values of TEAC were given as mmol Trolox for $1 \mathrm{~g}$ of olive leaf.

\subsection{High performance liquid chromatography (HPLC) analysis}

The HPLC analysis given in the literature [10] was used for the quantification of oleuropein and rutin. The HPLC equipment used was a Hewlett-Packard Series HP 1100 equipped with a diode array detector. The stationary phase was a C18 LiChrospher 100 analytical column $(250 \mathrm{~mm} \times 4 \mathrm{~mm}$ i.d. $)$ with a particle size of $5 \mathrm{~mm}$ thermostated at $30^{\circ} \mathrm{C}$. The flow rate was $1 \mathrm{ml} \mathrm{min}^{-1}$ and the absorbance changes were monitored at $280 \mathrm{~nm}$. The mobile phases for chromatographic analysis were: (A) acetic acid/water (2.5:97.5) and (B) acetonitrile. A linear gradient was run from $95 \%$ (A) and 5\% (B) to $75 \%$ (A) and $25 \%$ (B) during $20 \mathrm{~min}$; it changed to $50 \%$ (A) and (B) in $20 \mathrm{~min}$ (40 min, total time); in $10 \mathrm{~min}$ it changed to $20 \%$ (A) and $80 \%$ (B) (50 min, total time), after reequilibration in $10 \mathrm{~min}$ ( $60 \mathrm{~min}$, total time) to initial composition. Oleuropein and rutin in olive leaf extracts were identified by comparison of their retention times with the corresponding standards. Coumarin was used as internal standard for the quantification of oleuropein and rutin. Other standards were used only for identification of these compounds in olive leaf extracts.

\subsection{Adsorption and desorption of olive leaf antioxidants by using column system}

Crude olive leaf extract samples as $1 \mathrm{~g}$ and $1.5 \mathrm{~g}$ were dissolved in $40 \mathrm{ml}$ of deionized water at room temperature with magnetic stirring. Then, solutions were centrifuged at $5000 \mathrm{rpm}$ for $5 \mathrm{~min}$. Remaining water-soluble fractions in pellet were tried to be solved in further $5 \mathrm{ml}$ of deionized water and solution was centrifuged. The supernatants were collected and completed to $50 \mathrm{ml}$ with deionized water. A syringe column of $63 \mathrm{~mm}$ in length and $10 \mathrm{~mm}$ internal diameter with Teflon fittings was used. After filling it with $0.2 \mathrm{~g}$ of powdered silk fibroin, the column was preconditioned by washing with $5 \mathrm{ml}$ of deionized water, $\mathrm{EtOH}$ and deionized water, respectively. Then, $1 \mathrm{ml}$ of crude extract solution was loaded to the preconditioned column with constant flow rate with Gilson ${ }^{\mathrm{TM}}$ ASPEC XL liquid handling system. The loading breakthrough curves of oleuropein and rutin were obtained by analyzing their concentration at the column outlet with HPLC. After saturation of column with oleuropein and rutin, the fractions were eluted by washing the column with water and then with aqueous EtOH solutions. These fractions were analyzed by using HPLC.
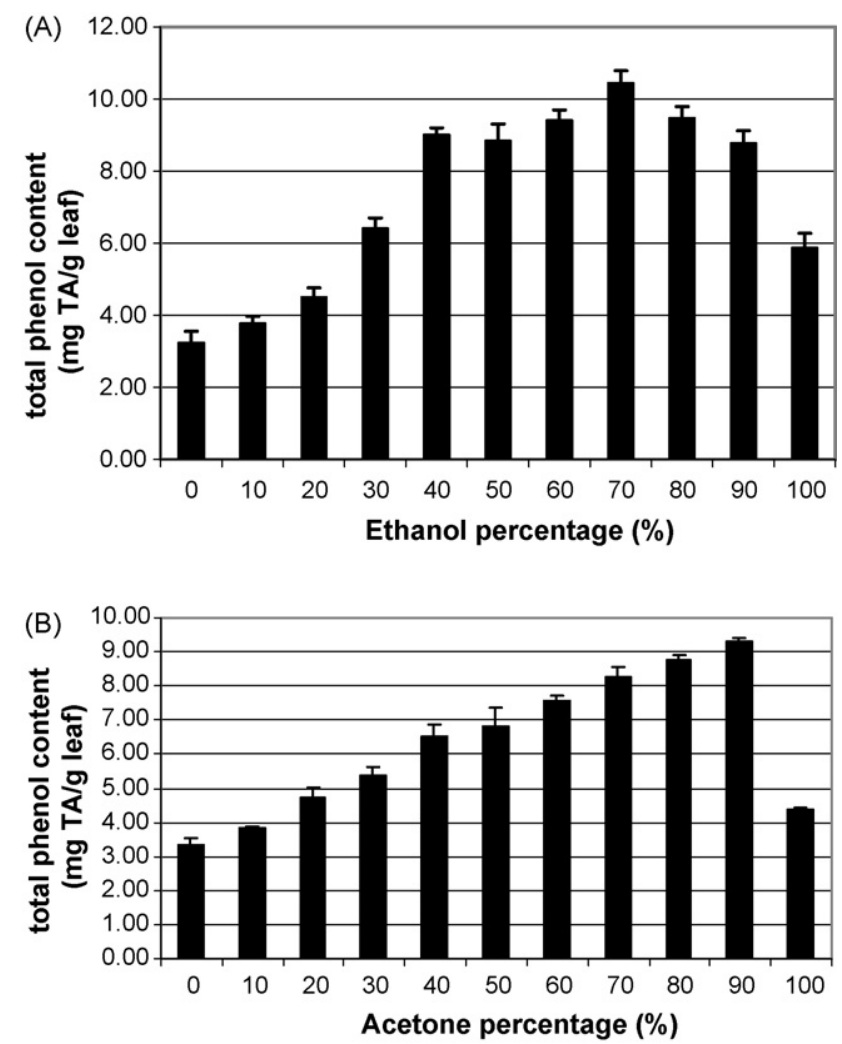

Fig. 1. Effect of extraction solvents on total phenol content of olive leaf extracts: (A) ethanol-water solution; (B) acetone-water solution.

\subsection{Data analysis}

Data were analyzed by using MINITAB Statistical Software, Release 14 for Windows. Data belongs to the analysis of the solvent effect on total phenol content were expressed as mean \pm standard deviation (S.D.). Any significant differences between solvents and samples were determined by one-way ANOVA followed by the Tukey test for multiple comparison considering differences statistically significant at $P<0.05$.

\section{Results and discussion}

\subsection{Extraction of olive leaf polyphenols}

The selection of extraction solvent is an important criterion for extracting desired components from plant materials. In this study two solvents and their aqueous forms were used to extract phenolics from olive leaves. The total phenol content of olive leaf extract in different extraction solvent systems was compared. Fig. 1 illustrates the effect of ethanol-water and acetone-water solvent system on extraction yield in terms of total phenol content. The highest phenol content was obtained with $70 \%$ aqueous ethanol solution as $10.10 \%$ and $90 \%$ aqueous acetone solution as 9.38 in terms of mg tannic acid per gram leaf. The total phenol content in $70 \%$ aqueous ethanol solution was slightly higher than the total phenol content in $90 \%$ aqueous acetone solution. However, ethanol is more preferable solvent because of its nontoxic, environmentally safe and inexpensive features. Ethanol alone 


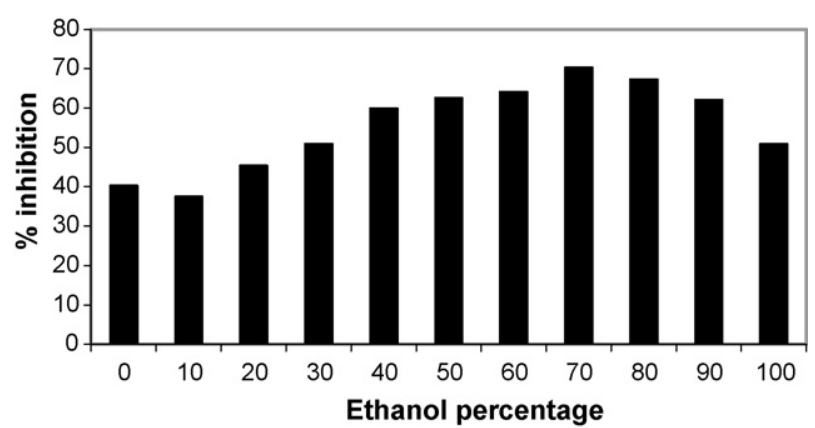

Fig. 2. Effect of solvent type on \% inhibition of ABTS radical cation.

or acetone alone was not effective as a solvent for extraction of phenolic compounds. These results revealed that water has important role in extraction process by increasing the diffusion of extractable polyphenols through the plant tissues.

Besides total phenol content, total antioxidant activity measurements were used in order to determine the effects of solvent type on the quality of extracts. The change in ethanol concentration affects the $\%$ inhibition of ABTS radical cation (Fig. 2). The highest $\%$ inhibition was observed with $70 \%$ aqueous ethanol extracts. This highest \% inhibition of $70 \%$ aqueous ethanol extracts can be explained by their high polyphenol contents as shown in Fig. 1A.

Fig. 3 shows the linear correlation between the total phenol content and antioxidant capacities of extracts obtained by different ethanol concentrations with high regression coefficient as 0.968 . This parallel relationship proves the total phenol content directly affects the antioxidative activity of olive leaf extract.

For the characterization of olive leaf extracts, quantification of major polyphenolic compounds, oleuropein and rutin present in olive leaf extracts were determined by using HPLC analyzes. Oleuropein and rutin were firstly identified by comparing their retention times with the corresponding standards and then their amounts in the extracts were quantified by external and internal calibration methods. Extraction was performed in $100 \%$, $70 \%, 50 \%, 30 \%, 20 \%$ aqueous ethanol solutions and $90 \%$ aqueous acetone solution to see whether the total phenol content is directly related with the oleuropein and rutin amounts. Moreover, extraction was done with water alone at $70^{\circ} \mathrm{C}$ and $40^{\circ} \mathrm{C}$ to see the effect of temperature on both oleuropein and rutin amounts and total antioxidant capacity.

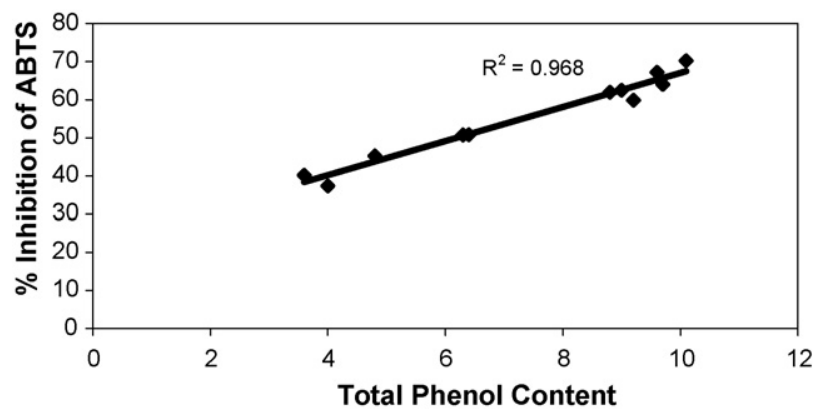

Fig. 3. Linear correlation between total phenol content of ethanolic extracts and their corresponding \% inhibition of ABTS.
Table 1

Antioxidant activities of olive leaf extracts obtained with different media

\begin{tabular}{ll}
\hline Sample & $\begin{array}{l}\text { TEAC }(\mathrm{mmol} \text { Trolox/g } \\
\text { olive leaf })\end{array}$ \\
\hline Olive leaf in $70 \%$ aqueous ethanol solution & 4.36 \\
Olive leaf in $50 \%$ aqueous ethanol solution & 2.68 \\
Olive leaf in $70^{\circ} \mathrm{C}$ water & 1.80 \\
\hline
\end{tabular}

Fig. 4 shows the effect of solvent type and ethanol percentage on the extraction yield of oleuropein and rutin, which is defined as mg extracted oleuropein, or rutin per gram of olive leaf in this study. The highest yield of oleuropein and rutin extraction was obtained with $70 \%$ aqueous ethanol solution as expected from the results of total phenol content analyses and antioxidant analyses. On the other hand, the extracted oleuropein amount was closer to each other with 50\% aqueous ethanol solution and $70^{\circ} \mathrm{C}$ water whereas the extraction efficiency was lower with $20 \%$ aqueous ethanol solution and water at $40^{\circ} \mathrm{C}$. Increasing the temperature of water highly affected the extraction yield and its extraction capacity was as nearly the same as with $50 \%$ aqueous ethanol solution.

Although the highest extraction yield was obtained with 70\% aqueous ethanol solution, the extraction efficiency of 50\% aqueous ethanol solution and water at $70^{\circ} \mathrm{C}$ were also comparable. However, high temperatures may cause the decomposition of oleuropein and rutin. For this reason, before choosing the extraction medium, the antioxidant capacities of samples in these three favorable conditions were analyzed by free radical scavenging method.

Table 1 shows that the highest antioxidant capacity was observed with $70 \%$ aqueous ethanol solution. The antioxidant capacity of extracting media decreased due to decreasing oleuropein and rutin contents, which prove that oleuropein and rutin have significant effect in olive leaf bioactivity. Although the oleuropein content of $50 \%$ aqueous ethanol solution and water at $70^{\circ} \mathrm{C}$ were closer to each other, their antioxidant capacities were significantly different. So it can be concluded that, increase in temperature decreased the antioxidant capacity of olive leave extracts. HPLC, antioxidant and total phenol content analyses showed that $70 \%$ aqueous ethanol solution was a promising medium to obtain a highly bioactive olive leaf extract.

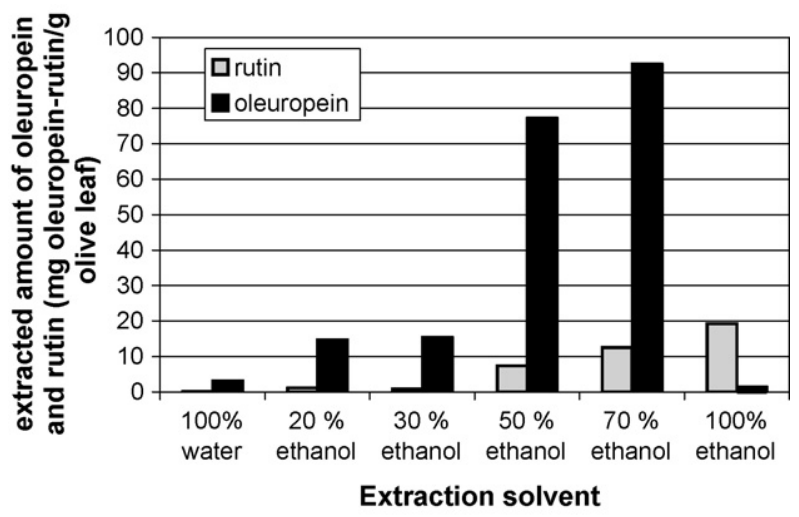

Fig. 4. Effect of solvent on extracted amount of oleuropein and rutin per gram of olive leaf. 

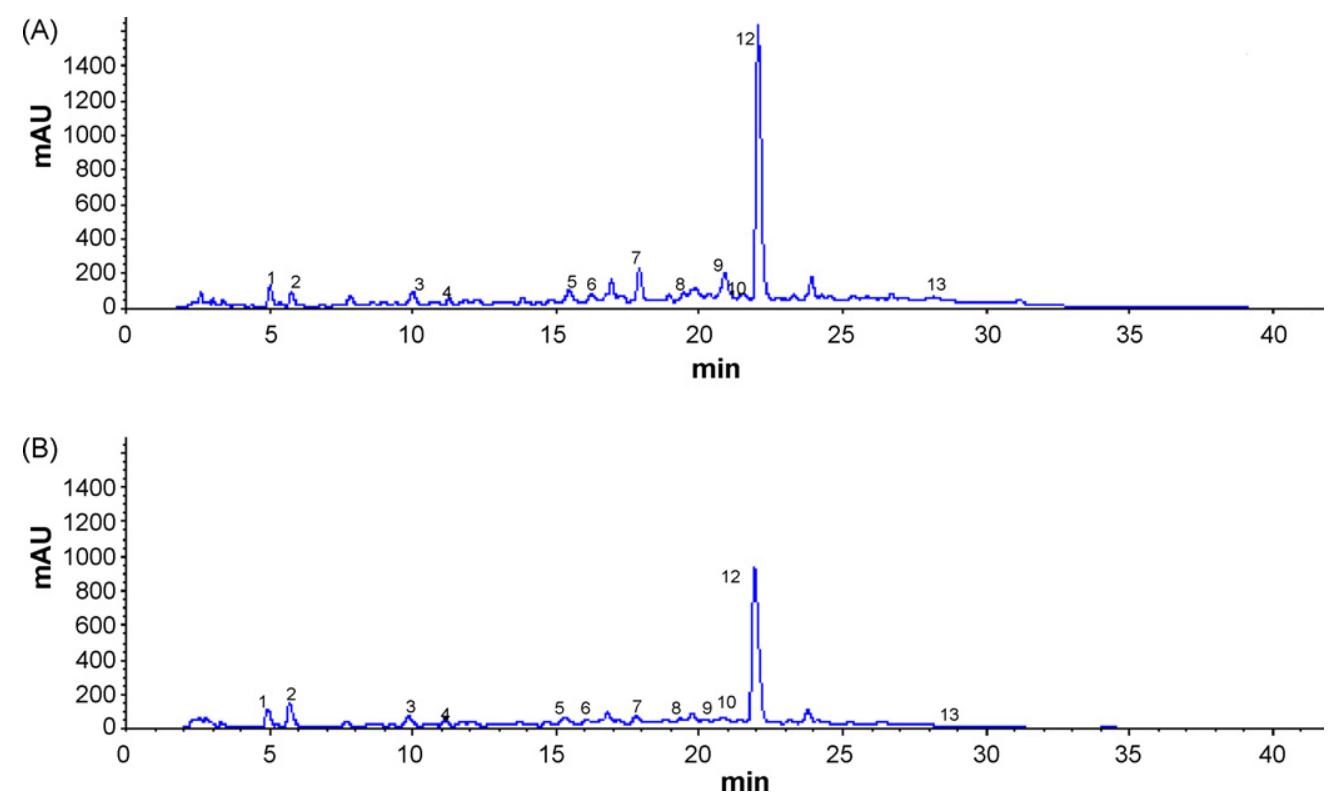

Fig. 5. HPLC profile of olive leaf crude extracts at two initial concentrations as (A) $3 \%$ OLE, w/v; (B) $2 \%$ OLE, w/v.

Then, dried crude olive leaf extracts were obtained with freeze drying after extraction with $70 \%$ aqueous ethanol solution. The efficiency of the extraction was increased with four successive extractions. The yield of extraction from olive leaf after four successive extractions was calculated as $52 \%$ whereas the yield of first extraction was calculated as $46 \%$. As a result, first extraction provided most of the extractable amount of product and there was no need to use extra solvents for successive extractions.

The HPLC and antioxidant analyses of freeze dried olive leaf extract were performed. Typical chromatograms of crude extract solutions at two different concentrations are given in Fig. 5. The HPLC profile showed several peaks corresponding to different major olive leaf polyphenols which were identified as hydroxytyrosol, tyrosol, rutin, luteolin-7-glucoside, verbascoside, apigenin-7-glucoside, oleuropein, luteolin. The numbered peaks and abundance of the main compounds in olive leaf extract were given in Table 2. The oleuropein and rutin content of $1-\mathrm{g}$

Table 2

Peak number and abundance (\% peak area) of the main phenolic compounds present in olive leaf crude extract solution ( $2 \%$ OLE, w/v)

\begin{tabular}{lcc}
\hline Phenolics & \# Of peak & \% Peak area \\
\hline Hydroxytyrosol & 1 & 2.27 \\
Tyrosol & 2 & 1.85 \\
Catechin & 3 & 2.23 \\
Caffeic acid & 4 & 1.09 \\
Vanillic acid & 5 & 3.076 \\
Vanillin & 6 & 2.519 \\
Rutin & 7 & 4.66 \\
Luteolin-7-glucoside & 8 & 1.92 \\
Verbascoside & 9 & 6.1 \\
Apigenin-7-glucoside & 10 & 2.3 \\
Diosmetin-7-glucoside & 11 & - \\
Oleuropein & 12 & 29 \\
Luteolin & 13 & 0.8 \\
\hline
\end{tabular}

olive leaf extract was found as $134.4 \mathrm{mg}$ and $18.3 \mathrm{mg}$, respectively so the percentages of oleuropein and rutin in olive leaf extract were $13.44 \%$ and $0.18 \%$, respectively. Some researchers investigated the oleuropein and rutin content of 14 cultivated olive tree leaves and they reported that the oleuropein content changed from $9.27 \%$ to $13.43 \%$ whereas rutin content changed from $0.07 \%$ to $0.35 \%$ depending on the type of the trees [11]. The antioxidant analysis of olive leaf extract was also performed and its antioxidant capacity was determined as $7.52 \mathrm{mmol}$ of TEAC/g olive leaf extract.

\subsection{Adsorption and desorption of olive leaf antioxidants by using a column system}

In our previous study batch isotherms have shown that silk fibroin is efficient adsorbent for purification of polyphenols from olive leaf extract [34]. To explore the adsorption capacity during continuous operation, a laboratory mini column filled with silk fibroin was used. The ratio $\left(C / C_{\mathrm{i}}\right)$ of the effluent concentration $C$ to the input concentration $C_{\mathrm{i}}$ was plotted against time to obtain the breakthrough curve at constant flow rate.

\subsubsection{Column loading}

Crude olive leaf extracts were loaded to the column filled with silk fibroin. Saturation of column with oleuropein and rutin was tried to be obtained by loading $1 \mathrm{ml}$ of crude extract subsequently. Column outlet solution was analyzed with HPLC for their oleuropein and rutin content. In order to evaluate the adsorption phenomena of oleuropein and rutin on the silk fibroin as solid phase, the effect of loading solution's concentration was investigated, using breakthrough curves. The obtained breakthrough curves are given in Figs. 6 and 7 for oleuropein and rutin, respectively. The selective accumulation of rutin and oleuropein on the hydrophobic silk fibroin revealed the presence of interaction between silk fibroin and polyphenols during the adsorption 


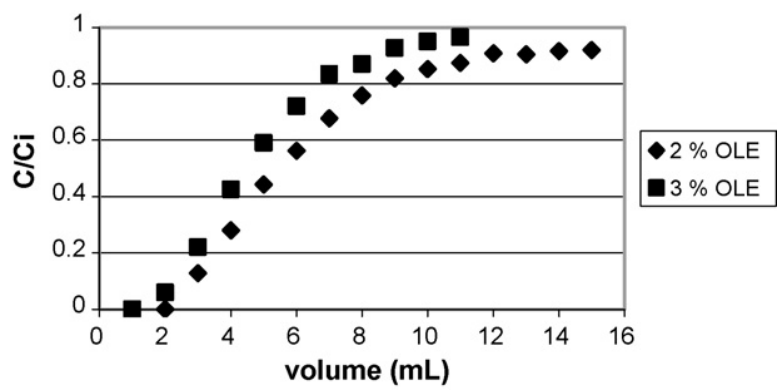

Fig. 6. Breakthrough profiles for oleuropein at different concentrations of loading solutions ( $2 \%$ OLE, w/v and $3 \%$ OLE, w/v of loading solutions).

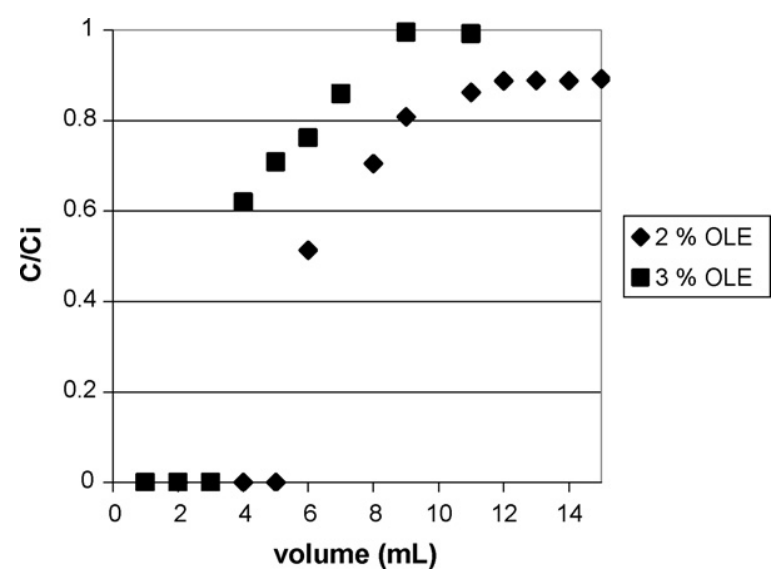

Fig. 7. Breakthrough profiles for rutin at different concentrations of loading solutions ( $2 \%$ OLE, w/v and 3\% OLE, w/v of loading solutions).

process. The other polyphenols in olive leaf extract such as verbascoside, apigenin-7-glucoside, luteolin-7-glucoside which have closer retention times to retention time of rutin and oleuropein, were also adsorbed on silk fibroin. However, the amount of these antioxidants in olive leaf extract and the adsorbed amount of them on silk fibroin are less compared to the adsorbed amount of oleuropein and rutin.

The comparison of HPLC chromatograms given in Fig. 8A and $\mathrm{B}$ indicated that, initially both oleuropein and rutin were rapidly adsorbed on the silk fibroin from initial extract solution. The first effluent was almost free of them as seen from
Table 3

Amount of adsorbed oleuropein and rutin $(q)$ on silk fibroin at different concentrations of loading solutions

\begin{tabular}{lrrrr}
\hline & Oleuropein & \multicolumn{3}{c}{ Rutin } \\
\hline$C_{\mathrm{i}\left(\mathrm{g}^{-1}\right)}$ & 3.24 & 4.56 & 0.42 & 0.57 \\
$q\left(\mathrm{mg} \mathrm{g}^{-1}\right.$ silk fibroin $)$ & 96.41 & 101.17 & 15.48 & 12.09 \\
\hline
\end{tabular}

Fig. 8B. As the crude extract solution was further loaded to the column, the silk fibroin was progressively saturated with oleuropein and rutin, therefore the amounts of both polyphenols started to increase until the saturation of silk fibroin was achieved (Fig. 8C and D).

Amount of adsorbed polyphenol $(q)$ was calculated as follows:

$q=\frac{\sum_{o=1}^{n} C_{o}-n C_{\mathrm{i}}}{w}$

where $C_{\mathrm{i}}$ denotes the initial concentration of the polyphenol in the aqueous olive leaf extract solution, $n$ is the number of loading of solution ( $1 \mathrm{ml}$ volume of each) and $w$ is the dry weight of the silk fibroin as adsorbent. The results obtained by this formula are listed in Table 3. It is clearly seen that, amount of adsorbed oleuropein on the silk fibroin increases with increasing initial concentration of loading solution. This situation is attributed to the existence of a dynamic equilibrium in the column system. However, in case of the rutin, adsorbed amount of rutin decreases with increasing initial concentration of rutin in loading solution.

\subsubsection{Eluting fractions}

After reaching to the saturation of column, elutions of different fractions were performed first with deionized water and then $40 \%$ aqueous ethanol solution. HPLC results of eluted fractions with deionized water are given in Table 4. Ten milliliters of water was enough to elute all oleuropein from the column. It can be clearly seen that purity of oleuropein increases. The other remaining fractions were eluted with $40 \% \mathrm{EtOH}$ solution from the silk fibroin filled column. Based on the sharp peaks at $18 \mathrm{~min}, 20 \mathrm{~min}$ and $21 \mathrm{~min}$ at chromatogram, it can be concluded that several other components found in crude extract solution are strongly adsorbed on silk fibroin. These peaks belong to the rutin,

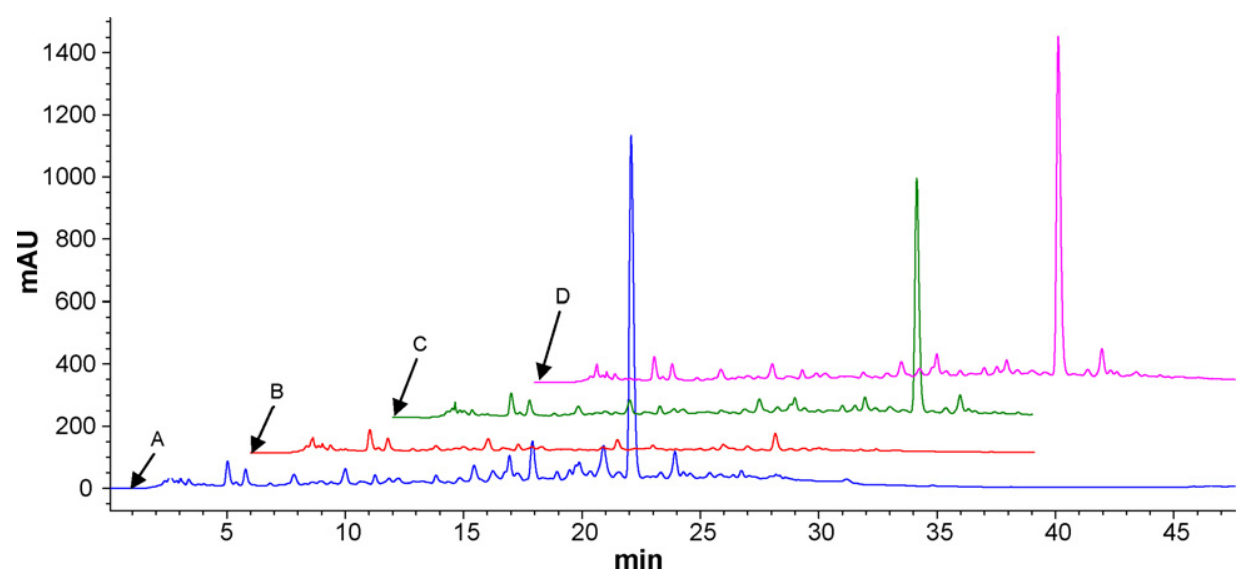

Fig. 8. HPLC chromatogram of (A) initial solution; (B) 1st effluent; (C) 8th effluent; (D) 12th effluent from the silk fibroin loaded column. 
Table 4

HPLC profile of fractions eluted from column with water and $40 \% \mathrm{EtOH}$ solution

\begin{tabular}{lcll}
\hline Phenolics & \# Of peak & $\begin{array}{l}\text { Water elution } \\
\% \text { Peak area }\end{array}$ & $\begin{array}{l}40 \% \text { EtOH elution } \\
\% \text { Peak area }\end{array}$ \\
\hline Hydroxytyrosol & 1 & - & - \\
Tyrosol & 2 & - & - \\
Catechin & 3 & - & - \\
Caffeic acid & 4 & - & - \\
Vanillic acid & 5 & - & 0.26 \\
Vanillin & 6 & 4 & - \\
Rutin & 7 & - & 13.84 \\
Luteolin-7-glucoside & 8 & - & 4.29 \\
Verbascoside & 9 & - & 15.46 \\
Apigenin-7-glucoside & 10 & 2.3 & 2.99 \\
Diosmetin-7-glucoside & 11 & - & - \\
Oleuropein & 12 & 62 & - \\
Luteolin & 13 & - & 7 \\
\hline
\end{tabular}

luteolein-7-glucoside and verbascoside, respectively (Table 4). As known from the literature, the most active flavonoids, rutin, catechin and luteolin had antioxidant activities almost 2.5 times those of vitamins $\mathrm{C}$ and $\mathrm{E}$ [10]. All of these polyphenols which possess high antioxidative activities increase the antioxidant capacity of olive leaf extract.

\section{Conclusion}

This study showed the importance of extraction solvent type on the extraction efficiency of polyphenolic compounds from olive leaves. The maximum recovery of bioactive compounds should be achieved in order to decrease the cost of further purification. In this study, $13.44 \%$ oleuropein and $0.183 \%$ rutin containing olive leaf extract was obtained. We explored the potential use of silk fibroin as an adsorbent for the recovery of polyphenols from the crude olive leaf extracts. Oleuropein is eluted from the silk fibroin filled column with high purity. At the same time fraction rich in rutin, luteolin-7-glucoside, verbascoside, apigenin-7-glucoside was obtained. This technique allows the production of antioxidant enriched olive leaf extracts.

\section{Acknowledgements}

Authors are grateful for financial support from the Turkish State Planning Organization (2002 K 120390) and Natural Products Research Development Unit (NPRDU/DUAG-002) located in Izmir Technology Development Zone and Izmir Institute of Technology (2006 IYTE BAP-27).

\section{References}

[1] H.R. Tang, A.D. Covington, R.A. Hancock, Biopolymers 70 (2003) 403.

[2] Y.F. Chu, J. Sun, X. Wu, R.H. Liu, J. Agric. Food Chem. 51 (2002) 6910.

[3] M.M. Cowan, Clin. Microbiol. Rev. 12 (1999) 564.
[4] J. Sun, Y.F. Chu, X. Wu, R.H. Liu, J. Agric. Food Chem. 50 (2002) 7449.

[5] R. Bouhamidi, V. Prevost, A. Nouvelot, Plant Biology and Pathalogy: Comptes Rendus de l'Académie des Sciences - Series III - Sciences de la Vie 321(1998) 31.

[6] R.H. Liu, Am. J. Clin. Nutr. 78 (3S) (2003) 517S.

[7] R.H. Liu, J. Nutr. 134 (12S) (2004) 3479S.

[8] D. Ryan, H. Lawrence, P.D. Prenzler, M. Antolovich, K. Robards, Anal. Chim. Acta 445 (2001) 67.

[9] I.C.F.R. Ferreira, L. Barros, M.E. Soares, M.L. Bastos, J.A. Pereira, Food Chem. 103 (2007) 188

[10] O.B. Garcia, J. Castillo, J. Lorente, A. Ortuno, J.A. Del Rio, Food Chem. 68 (2000) 457.

[11] C. Savournin, B. Baghdikian, R. Elias, F. Dargouth-Kesraoui, K. Boukef, G. Balansard, J. Agric. Food Chem. 49 (2001) 618.

[12] E. Campeol, G. Flamini, P.L. Cioni, I. Morelli, R. Cremonini, L. Ceccarini, J. Agric. Food Chem. 51 (2003) 1994.

[13] S. Lee-Huang, P.L. Huang, D. Zhang, J.W. Lee, J. Bao, Y. Sun, Y.T. Chang, J.Z.H. Zhang, P.L. Huang, Biochem. Biophys. Res. Commun. 354 (2007) 872.

[14] J. Bao, D.W. Zhang, J.Z.H. Zhang, P.L. Huang, P.L. Huang, S. Lee-Huang, FEBS Lett. 581 (2007) 2737.

[15] G. Flamini, P.L. Cioni, I. Morelli, J. Agric. Food Chem. 51 (2003) 1382.

[16] A. Ranalli, S. Contento, L. Lucera, M. Febo, D. Archegiani, V. Fonzo, J. Agric. Food Chem. 54 (2006) 434.

[17] S. Silva, L. Gomes, F. Leitão, A.V. Coelho, L. Vilas Boas, Food Sci. Technol. Int. 12 (2006) 385.

[18] J. Tabera, A. Guinda, A. Ruiz-Rodrigez, F.J. Senorans, E. Ibanez, T. Albi, G. Reglero, J. Agric. Food Chem. 52 (2004) 4774.

[19] R. Japón-Luján, J.M. Luque-Rodríguez, M.D. Luque de Castro, J. Chromatogr. A 1108 (2006) 76.

[20] R. Japón-Luján, M.D. Luque de Castro, J. Chromatogr. A 1136 (2006) 185.

[21] F. Paiva-Martins, R. Correia, S. Felix, P. Ferreira, M.H. Gordon, J. Agric. Food Chem. 55 (2007) 4139.

[22] S.Y. Yoon, W.J. Choi, J.M. Park, J.W. Yang, Biotechnol. Techn. 11 (1997) 553.

[23] E. Aehle, S. Raynaud-Le Grandic, R. Ralainirina, S. Baltora-Rosset, F. Mesnard, C. Prouillet, J.C. Maziere, M.A. Fliniaux, Food Chem. 86 (2004) 579.

[24] M.H.L. Ribeiro, D. Silveira, S. Ferreira-Dias, Eur. Food Res. Technol. 215 (2002) 462

[25] A. Kraemer-Schafhalter, H. Fuchs, W. Pfannhauser, J. Sci. Food Agric. 78 (1998) 435.

[26] M. Scordino, A. Di Mauro, A. Passerini, E. Maccarone, Adsorption of flavonoids on resins: hesperidin, J. Agric. Food Chem. 51 (2003) 6998-7004.

[27] M. Scordino, A. Di Mauro, A. Passerini, E. Maccarone, J. Agric. Food Chem. 52 (2004) 1965.

[28] N. Seeram, R. Lee, M. Hardy, D. Heber, Sep. Purif. Technol. 41 (2005) 49.

[29] E.M. Silva, D.R. Pompeu, Y. Larondelle, H. Rogez, Sep. Purif. Technol. 53 (2007) 274.

[30] G.H. Altman, F. Diaz, C. Jakuba, T. Calabro, R.L. Horan, J. Chen, H. Lu, J. Richmond, D.L. Kaplan, Biomaterials 24 (2003) 401.

[31] O. Bayraktar, Ö. Malay, Y. Özgarip, A. Batıgün, Eur. J. Pharm. Biopharm. 60 (2005) 373.

[32] S.J. Park, K.Y. Lee, W.S. Ha, S.Y. Park, J. Appl. Polym. Sci. 74 (1999) 2571.

[33] P. Kongkachuichaya, A. Shitangkoonb, N. Chinwongamorn, Dyes Pigments 53 (2002) 179.

[34] D. Bayçın, E. Altıok, S. Ülkü, O. Bayraktar, J. Agric. Food Chem. 55 (2007) 1227. 